\title{
The surface layer integral method for the modelling of the radial gravity gradient component over sea surface
}

DOI: https://doi.org/10.1515/jogs-2019-0012

Received November 30, 2018; accepted July 19, 2019

\begin{abstract}
For the modelling and determination of the Earth's external gravity potential as well as its secondorder radial derivatives in the space near sea surface, the surface layer integral method was discussed in the paper. The reasons for the applicability of the method over sea surface were discussed. From the original integral formula of disturbing potential based on the surface layer method, the expression of the radial component of the gravity gradient tensor was derived. Furthermore, an identity relation was introduced to modify the formula in order to reduce the singularity problem. Numerical experiments carried out over the marine area of China show that, the modified surface layer integral method effectively improves the accuracy and reliability of the calculation of the secondorder radial gradient component of the disturbing potential near sea surface.
\end{abstract}

Keywords: disturbing potential; second order radial derivative; singularity; surface layer integral

\section{Introduction}

In geodesy and space research, the calculation of the external disturbing gravity field is of great importance (Moritz, 1966; Heiskanen and Moritz, 1967; Wenzel, 2005; Bettadpur, 2015). Currently there are three kinds of mathematical models for the computation of external disturbing gravity field. The first is the spherical harmonics series expression which is usually used as the basic model of remove and restore method. The second kind includes the direct integral model based on classical Stokes theory, the surface layer integral method and the upward continuation model

\footnotetext{
*Corresponding Author: D. Zhao: Surveying and Mapping Engineering Department, Geospatial Information College, Information Engineering University, Zhengzhou Science Avenue 1, Henan Province, China, 450001, E-mail: zhaodongming0510@126.com

Z. Gong, J. Feng: Surveying and Mapping Engineering Department, Geospatial Information College, Information Engineering University, Zhengzhou Science Avenue 1, Henan Province, China, 450001
}

based on Poisson integral, which are characterized by using gravity observations on the Earth's surface directly and singularity problem when the computation point is close to the surface. The third kind are developed from Bjerhammar theory, which includes methods like point mass method, etc.

Due to the simple kernel and convenient input data source, say, gravity observations and height anomalies, the surface layer integral method is more suitable for the calculation of external disturbing gravity field over sea surface (Heck, 2003). The only defect of the surface layer method (or common to similar integral methods) is the singularity problem when the calculation point is close to the sea surface. In literatures there are effective solutions to the singularity problem (Heiskanen and Moritz, 1967; Bian, 1997; Hwang et al., 1998; Guo \& Xu, 2011) in the computation of external gravity elements.

Due to the fact that the vertical gradient of disturbing gravity plays an important role in physical geodesy as well as geophysics, here in the paper the calculation of the second order radial derivative of disturbing potential using surface layer integral method is proposed. The singularity problem in its computation is discussed and improvement was made to the integral formula to reduce the singularity effect. Numerical experiments were also made to test the modified method.

\section{The Surface Layer Integral Method for the Second-order Radial Derivative of Disturbing Potential}

From Heiskanen and Moritz (1967), the surface layer integral method can be used to compute the external disturbing potential as well as its first and second order radial 
derivatives, which are expressed in Eq. (1) (3).

$$
\begin{gathered}
T_{P}=\frac{R^{2}}{2 \pi} \iint_{\sigma} \frac{\mu}{l} d \sigma \\
\delta g_{r}=-\frac{\partial T}{\partial r}=\frac{R^{2}}{2 \pi} \iint_{\sigma}\left(\frac{3(r-R \cos \psi)}{l^{3}}\right) d \sigma \\
\delta g_{r r}=\frac{\partial^{2} T}{\partial r^{2}}=\frac{R^{2}}{2 \pi} \iint_{\sigma} \mu\left(\frac{3(r-R \cos \psi)^{3}}{l^{5}}-\frac{1}{l^{3}}\right) d \sigma
\end{gathered}
$$

where

$$
\begin{gathered}
\mu=\Delta g+\frac{3 T}{2 R}=\Delta g+\frac{3 y}{2 R} \zeta \\
l=\sqrt{r^{2}+R^{2}-2 r R \cos \psi} \\
\cos \psi=\sin \varphi \sin \varphi^{\prime}+\cos \varphi \cos \varphi^{\prime} \cos \left(\lambda^{\prime}-\lambda\right) \\
r=R+h
\end{gathered}
$$

It should be pointed out that Eqs. (2) (3) hold for the computation point of the integration in the exterior of the sphere of radius $R$, i.e. $h>0$.

In the above formulas, $T_{P}$ is the disturbing potential of the computation point $\mathrm{P} ;(r, \phi, \lambda)$ and $\left(R, \phi^{\prime}, \lambda^{\prime}\right)$ represents the geocentric radius, geocentric latitude and longitude of the computation point and flow point on the sphere respectively; $\psi$ is the spherical angle between the computation point and the moving point; $l$ is the distance between the computation point and the moving point; $\Delta g$ is the free-air gravity anomaly on surface; $T$ is the disturbing potential at the surface point; $\zeta$ is the height anomaly at the surface point (which equals $T / y$ in the context of Molodensky's theory); $y$ is the normal gravity at the surface point; $\mu$ is called the surface density; $R$ is the mean radius of the Earth; $h$ is geodetic height; $d \sigma$ is the area element of unit sphere integral.

\section{Non-singular Surface Layer Integral Method for the Second-order Radial Derivative of Disturbing Potential}

From Eq. (1) (7), when the height of the computation point $h \rightarrow 0$ and the spherical angle $\psi \rightarrow 0$, singularity problems arise for the numerical integration of Eq. (2) and Eq. (3). To overcome the singularity problem in handling Eq. (3), here an identity relation according to the theory of the radial derivative of harmonic function from Heiskanen and Moritz (1967) is introduced and convert the original integral model into continuous model with stable numerical solutions. The identity deals with the integral of the derivative of the reciprocal of the distance between the computation point and the moving point with respect to radial distance.

Firstly, the following identity can be derived by double integration

$$
\begin{aligned}
\frac{R^{2}}{2 \pi} \mu_{P_{0}} & =R^{2} \mu_{P_{0}} \int_{0}^{\pi}\left(\frac{3(r-R \cos \psi)^{2}}{l^{5}}-\frac{1}{l^{3}}\right) \sin \psi d \psi \\
& =\frac{4 R^{2}}{r^{3}} \mu_{P_{0}}
\end{aligned}
$$

Where $\mu_{P_{0}}$ is the surface density at the surface computation point. From the subtraction of Eq. (3) and Eq. (8) the following relation is derived

$\delta g_{r r}=\frac{R^{2}}{2 \pi} \iint_{\sigma}\left(\mu-\mu_{P_{0}}\right)\left(\frac{3(r-R \cos \psi)^{2}}{l^{5}}-\frac{1}{l^{3}}\right)+\frac{4 R^{2}}{r^{3}} \mu_{P_{0}}$

In Eq. (9) the singularity is reduced and numerical continuity is achieved on the boundary surface. It should be pointed out that in practice, due to the limitations of data coverage, the global integration is always split into near-zone and far-zone parts. At the same time, a global gravity potential model that is truncated to some degree and order is introduced and in the meantime the removeand-restore technique is applied. Therefore, in the near zone the residual gravity from that of gravity potential model is used in computation, and in the far zone, the gravity potential model is directly used.

In the near-zone computations, as far as efficiency is concerned, the data coverage around the computation point is often divided into several ring-zone from near to far zones, and in the near ring-zone that is near the computation point the data resolution is higher than those in the ring-zones that is far from the computation point. For example, a combination of ring-zone with different data resolution that is applied is like $1^{\prime} \times 1^{\prime}, 5^{\prime} \times 5^{\prime}, 20^{\prime} \times 20^{\prime}$ and $1^{\circ} \times 1^{\circ}$ from near to far zones.

On the other hand, in the data coverage of the highest data resolution that contains the computation point, which is also a spherical cap, say, the $2^{\circ} \times 2^{\circ}$ data coverage in which the data resolution is $1^{\prime} \times 1^{\prime}$, special attention should be paid in handling. Assuming the integral radius for this spherical cap is $\psi_{0}$, the following integration is derived

$$
\begin{aligned}
& \frac{R^{2}}{2 \pi} \mu_{P_{0}} \int_{0}^{2 \pi} \int_{0}^{\psi_{0}}\left(\frac{3(r-R \cos \psi)^{2}}{l^{5}}-\frac{1}{l^{3}}\right) \sin \psi d \psi d \alpha \\
& =R^{2} \mu_{P_{0}} \int_{0}^{\psi_{0}}\left(\frac{3(r-R \cos \psi)^{2}}{l^{5}}-\frac{1}{l^{3}}\right) \sin \psi d \psi \\
& =\frac{3 r R^{3} \mu_{P_{0}}\left(r \cos ^{2} \psi_{0}-2 R \cos \psi_{0}+r\right)+2 R^{2}\left(R^{3}-r^{3} \cos \psi_{0}\right) \mu_{P_{0}}}{l_{\psi_{0}}^{3} r^{3}}+\frac{2 R^{2} \mu_{P_{0}}}{r^{3}}
\end{aligned}
$$


where $\alpha$ is the azimuth reckoned from north and

$$
\begin{gathered}
l_{\psi_{0}}=\sqrt{r^{2}+R^{2}-2 r R \cos \psi_{0}} \\
\cos \alpha=\frac{\cos \varphi \sin \varphi^{\prime}-\sin \varphi \cos \varphi^{\prime} \cos \left(\lambda^{\prime}-\lambda\right)}{\sin \psi} \\
\sin \alpha=\frac{\cos \varphi^{\prime} \sin \left(\lambda^{\prime}-\lambda\right)}{\sin \psi}
\end{gathered}
$$

Handled in a similar way to the derivation of Eq.(9), the following formula is derived

$$
\delta g_{r r}\left(1^{\prime}\right)=\frac{R^{2}}{2 \pi} \int_{0}^{2 \pi} \int_{0}^{\psi_{0}}\left(\mu-\mu_{P_{0}}\right)\left(\frac{3(r-R \cos \psi)^{2}}{l^{5}}-\frac{1}{l^{3}}\right)
$$

$\sin \psi d \psi d \alpha+$

$\frac{3 r R^{3}\left(r \cos ^{2} \psi_{0}-2 R \cos \psi_{0}+r\right) \mu_{P_{0}}+2 R^{2}\left(R^{3}-r^{3} \cos \psi_{0}\right) \mu_{P_{0}}}{l_{\psi_{0}}^{3} r^{3}}$ $+\frac{2 R^{2} \mu_{P_{0}}}{r^{3}}$

In Eq. (13) the singularity problem and data discontinuity problem were reduced. And therefore, in practical applications, the data coverage should just be divided into internal zone (corresponding to $1^{\prime} \times 1^{\prime}$ data resolution) and external zones (including coverage of $5^{\prime} \times 5^{\prime}, 20^{\prime} \times 20^{\prime}, 1^{\circ} \times 1^{\circ}$ data resolutions), and their coverage (Fig. 1) could be determined using the method discussed in (Heiskanen and Moritz, 1967), and then Eq. (13) is applied for the computation within internal zone, and Eq.(3) is applied for outside zones. The above-mentioned process is formulated as follows

$$
\begin{aligned}
\delta g_{r r}(\text { total })= & \delta g_{r r}\left(1^{\prime}\right)+\delta g_{r r}\left(5^{\prime}\right)+\delta g_{r r}\left(20^{\prime}\right)+\delta g_{r r}\left(1^{\circ}\right) \\
& +\delta g_{r r}(\text { Mod_trunc })
\end{aligned}
$$

In Eq. (14), $\delta g_{r r}\left(1^{\prime}\right)$ is computed using Eq. (13), and $\delta g_{r r}\left(5^{\prime}\right), \delta g_{r r}\left(20^{\prime}\right)$ and $\delta g_{r r}\left(1^{\circ}\right)$ should be computed using Eq. (3). $\delta g_{r r}($ Mod_trunc) stands for the contribution of global gravity potential model truncated to some max degree and order, which is formulated as

$$
\begin{aligned}
& \delta g_{r r}(\text { Mod_trunc })=\frac{G M}{r^{3}} \sum_{n=2}^{N}(n+1)(n+2)\left(\frac{R}{r}\right)^{n} \\
& \sum_{m=0}^{n}\left(\bar{C}_{n m}^{\star} \cos m \lambda+\bar{S}_{n m} \sin m \lambda\right) \bar{P}_{n m}(\sin \varphi)
\end{aligned}
$$

where $G M$ is the universal gravitation constant multiplied by the Earth's total mass; $\left(\bar{C}_{n m}^{\star}, \bar{S}_{n m}\right)$ is the fully normalized disturbing gravity potential coefficients; $\bar{P}_{n m}(\sin \varphi)$ is the fully normalized Legendre polynomial; $N$ is the highest degree of the spherical harmonic series expression.

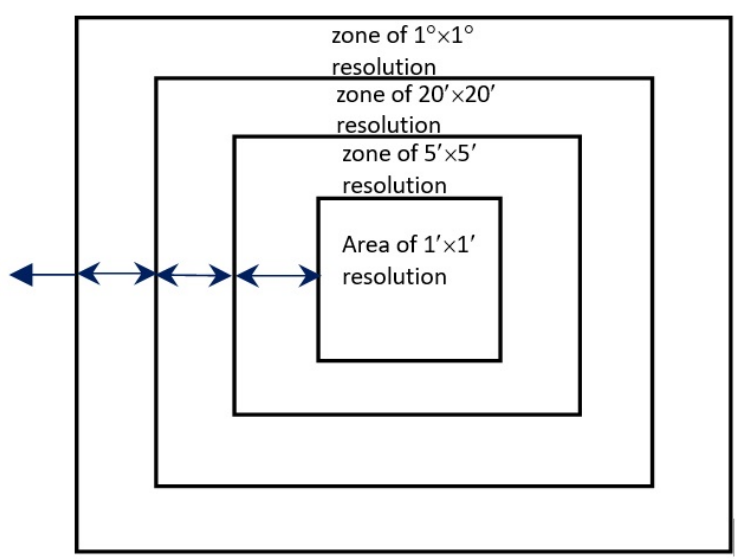

Fig. 1. Limits of the regions according to data resolutions

\section{Numerical Experiments}

An area that covers $30^{\circ} \mathrm{N} \sim 35^{\circ} \mathrm{N}$ and $120^{\circ} \mathrm{E} \sim 125^{\circ} \mathrm{E}$ was selected as the experiment area. In the area the gravity anomalies and height anomalies are of $1^{\prime} \times 1^{\prime}$ resolution and were generated using the gravity potential model EIGEN6C4 of which the max degree and order is 2190 . Within the coverage $31^{\circ} \mathrm{N} 34^{\circ} \mathrm{N}$ and $121^{\circ} \mathrm{E} \sim 124^{\circ} \mathrm{E}$ the reference values of the second-order radial gravity gradient of $1^{\prime} \times 1^{\prime}$ resolution were also generated using the gravity potential model EIGEN-6C4 at different height levels and each level contains 32400 points. Furthermore, the long wavelength part of the gravity field is represented using EIGEN-6C4 that is truncated to 360 degree and order.

The workflow of the numerical experiment is as follows. Within the experiment area, the second-order radial derivatives of disturbing potential are calculated in two ways. For the first way, the second-order radial derivative of disturbing potential is computed using the original integral Eq. (3) with input data being the model generated gravity anomalies, and for the second way, the secondorder radial derivative of disturbing potential is computed using Eq. (9) and Eq. (14). In either way, the computed second-order radial derivative of disturbing potential were compared with the reference values and statistics were obtained. In the experiment, grid intensification or resolution enhancement were also made so as to approach theoretical result. In the following discussions height means the height above the sea geoid.

(1) Height $h$ approaches 0

Assuming that the height $h$ approaches 0 and here it is set $h=0.2 \mathrm{~m}$. Due to the singularity of the kernel, the result of the original integral method using Eq. (3) is really bad enough, as is shown in Table 1. However, if the grid reso- 
Table 1. Result of Original Method /Unit: E (Eötvös $\left.=10^{-9} \mathrm{~s}^{-2}\right)$

\begin{tabular}{ccccc}
\hline $\begin{array}{c}\text { Intensification } \\
\text { Point Count }\end{array}$ & MIN & MAX & MEAN & STD \\
\hline $60 \times 60$ & $-1.210 \mathrm{E}+11$ & $1.777 \mathrm{E}+11$ & $-1.393 \mathrm{E}+08$ & $7.670 \mathrm{E}+09$ \\
$120 \times 120$ & $-3.025 \mathrm{E}+10$ & $4.444 \mathrm{E}+10$ & $-3.483 \mathrm{E}+07$ & $1.917 \mathrm{E}+09$ \\
$240 \times 240$ & $-7.562 \mathrm{E}+09$ & $1.111 \mathrm{E}+10$ & $-8.707 \mathrm{E}+06$ & $4.794 \mathrm{E}+08$ \\
$480 \times 480$ & $-1.890 \mathrm{E}+09$ & $2.777 \mathrm{E}+09$ & $-2.177 \mathrm{E}+06$ & $1.198 \mathrm{E}+08$ \\
$960 \times 960$ & $-4.724 \mathrm{E}+08$ & $6.940 \mathrm{E}+08$ & $-5.441 \mathrm{E}+05$ & $2.992 \mathrm{E}+07$ \\
\hline
\end{tabular}

Table 2. Results of Improved Method /Unit: E

\begin{tabular}{ccccc}
\hline $\begin{array}{c}\text { Intensification } \\
\text { Point Count }\end{array}$ & MIN & MAX & MEAN & STD \\
\hline $60 \times 60$ & -14.2995 & 16.9906 & 0.0043 & 2.7738 \\
$120 \times 120$ & -14.2760 & 16.9843 & 0.0043 & 2.7735 \\
\hline
\end{tabular}

lution is increased, the mean difference and standard difference becomes smaller, which is caused by the increase of grid resolution.

In Table 2, the results show that the improved method using Eq. (9) (14) successfully reduced the singularity of the kernel. And the standard difference does not improve prominently with the increase of the intensification points. The main cause is that in the improved method $\mu-\mu_{P_{0}}$ were used and their values approach 0 .

(2) Height $h=200 \mathrm{~m}$

Set $h=200 \mathrm{~m}$ and on such occasion, the kernel is not singular. The results of the original method are shown in Table 3. With the increase of intensification point count, the mean difference and standard difference becomes smaller. The results of the improved method is shown in Table 4, which is better than those of the original method.

(3) $h=500 \mathrm{~m}, h=1000 \mathrm{~m}$ and $h=5000 \mathrm{~m}$

The variation of kernel becomes relatively smooth. However, it is found that the accuracy of the improved method becomes lower than that of the original method. And with the increasing of intensification point count, the accuracy decreases to some extent. Calculations were also made for $h=1000 \mathrm{~m}, h=5000 \mathrm{~m}$, and the result is similar to those of $h=500 \mathrm{~m}$. Such variation of accuracy can be attributed to not increasing the integral radius with height increasing.

\section{Summary}

In order to apply the surface layer integral method to calculate the second-order radial derivative of the disturbing potential, we developed a different way to deal with the integral due to the fact that there exist singularity and discontinuity problem in the original integral when the com- putation point approaches the moving point on the boundary. An identity is introduced to improve the original surface layer integral formula for calculation of the second order radial derivative of the disturbing potential. The identity starts from the theory of the radial derivative of a harmonic function from (Heiskanen \& Moritz, 1967), which deals with the integral of the derivative of the reciprocal of the distance between the computation point and the moving point with respect to radial distance. The newly derived integral thus reduces the singularity and the discontinuity problem of the original method. Numerical experiment revealed that the improved integral method can reduce the singularity effectively with height below $200 \mathrm{~m}$ and raise the accuracy of calculation prominently especially when the height approaches 0 , which means that the computation point nearly coincides with the moving point on the boundary. Results also revealed that with the increase of the height of computation point, when the height is above $200 \mathrm{~m}$, the accuracy of the improved integral method decreased a little compared to the original method. Nevertheless, the newly derived method is useful in the calculation of the second order radial derivative of disturbing potential especially when the computation point approaches the boundary. Future work will be focused on the details of the improved integral on data coverage and omission error analysis of using a truncated gravity potential model.

Acknowledgements: The work in the paper is financially supported by the Chinese National Natural Science Foundation project No. 41574020.

The author thanks the anonymous referees for their valuable advices to improve the quality of the present article. 
Table 3. Result of Original Method /Unit: E

\begin{tabular}{ccccc}
\hline $\begin{array}{c}\text { Intensification } \\
\text { Point Count }\end{array}$ & MIN & MAX & MEAN & \multicolumn{1}{l}{ STD } \\
\hline $60 \times 60$ & -5.0596 & 8.0337 & -0.0009 & 1.4470 \\
$120 \times 120$ & -3.5594 & 5.1326 & -0.0006 & 0.9129 \\
$240 \times 240$ & -2.8282 & 3.6913 & -0.0004 & 0.6600 \\
$480 \times 480$ & -2.4770 & 3.1598 & -0.0003 & 0.5424 \\
$960 \times 960$ & -2.3017 & 2.9101 & -0.0003 & 0.4875 \\
\hline
\end{tabular}

Table 4. Results of Improved Method /Unit: E

\begin{tabular}{ccccc}
\hline $\begin{array}{c}\text { Intensification } \\
\text { Point Count }\end{array}$ & MIN & MAX & MEAN & STD \\
\hline $60 \times 60$ & -2.4940 & 1.7727 & 0.0002 & 0.5089 \\
$120 \times 120$ & -2.4991 & 1.7783 & 0.0002 & 0.5083 \\
\hline
\end{tabular}

Table 5. Statistics of Results of the Original Method ( $h=5000 \mathrm{~m})$ Unit:/E

\begin{tabular}{ccccc}
\hline $\begin{array}{c}\text { Intensification } \\
\text { Point Count }\end{array}$ & MIN & MAX & MEAN & STD \\
\hline $60 \times 60$ & -0.2721 & 0.1711 & -0.0009 & 0.0461 \\
$120 \times 120$ & -0.2419 & 0.1652 & -0.0009 & 0.0399 \\
$240 \times 240$ & -0.2271 & 0.1670 & -0.0009 & 0.0371 \\
$480 \times 480$ & -0.2198 & 0.1679 & -0.0009 & 0.0358 \\
$960 \times 960$ & -0.2162 & 0.1684 & -0.0009 & 0.0352 \\
\hline
\end{tabular}

Table 6. Statistics of Results of the Improved Method ( $h=5000 \mathrm{~m})$ Unit: $/ \mathrm{E}$

\begin{tabular}{ccccc}
\hline $\begin{array}{c}\text { Intensification } \\
\text { Point Count }\end{array}$ & MIN & MAX & MEAN & STD \\
\hline $3 \times 3$ & -3.8603 & 2.5877 & -0.0004 & 0.7353 \\
$30 \times 30$ & -3.8712 & 2.5926 & -0.0004 & 0.7368 \\
$60 \times 60$ & -3.8721 & 2.5930 & -0.0004 & 0.7370 \\
$120 \times 120$ & -3.8726 & 2.5932 & -0.0004 & 0.7370 \\
\hline
\end{tabular}

Table 7. Statistics of Results ( $h=500 \mathrm{~m})$ Unit:/E

\begin{tabular}{llrlll}
\hline & $\begin{array}{l}\text { Intensification } \\
\text { Point Count }\end{array}$ & \multicolumn{1}{c}{ MIN } & MAX & MEAN & STD \\
\hline Original Method & $60 \times 60$ & 1.1265 & 1.6147 & -0.0010 & 0.3160 \\
Improved Method & $60 \times 60$ & -3.8151 & 2.5229 & -0.0004 & 0.7144 \\
\hline
\end{tabular}

Table 8. Statistics of Results ( $h=1000 \mathrm{~m})$ Unit:/E

\begin{tabular}{llclll}
\hline & $\begin{array}{c}\text { Intensification } \\
\text { Point Count }\end{array}$ & MIN & MAX & MEAN & STD \\
\hline Original Method & $60 \times 60$ & -1.9872 & 1.2746 & -0.0009 & 0.3480 \\
Improved Method & $60 \times 60$ & -4.4502 & 2.8314 & -0.0006 & 0.8079 \\
\hline
\end{tabular}

\section{References}

Bettadpur S., Ries J., Eanes R., Nagel P., Pie N., Poole S., Richter T. and Save H., 2015, Evaluation of the GGM05 Mean Earth Gravity models, Geo. Res. Abs., 17, EGU2015-4153.
Bian S. F., 1997, Some cubature formulas for singular integrals in physical geodesy. Journal of Geodesy, 71, 443-453.

Bjerhammar A., 1987, Discrete Physical Geodesy. Rep No. 380, Dept. of Geodetic Science and Surveying, The Ohio State University, Columbus. 
Förste C., Bruinsma S.L., Abrikosov O., Lemoine J. M., Marty J.C., Flechtner F., Balmino G., Barthelmes F. and Biancale R., 2015,EIGEN-6C4 The latest combined global gravity field model including GOCE data up to degree and order 2190 of GFZ Potsdam andGRGS Toulouse. http://dx.doi.org/10.5880/icgem.2015.1

Gatti A., Reguzzoni M., Migliaccio F. and Sanso F., 2014, Space-wise grids of gravity gradients from GOCE data at nominal satellite altitude.[GOCE User Workshop]. Paris

Goli M., Najafi-Alamdari M., 2011, Planar, spherical and ellipsoidal approximations of Poisson's integral in near zone. Journal of Geodetic Science, 1, 1, 17-24.

Guo D. M. and Xu H., 2011, Study on singular integral of local terrain corrections, Chinese Journal of Geophysics, 54(4):977-983

Heck B., 2003, Integral Equation Methods in Physical Geodesy. In: Grafarend E.W., Krumm F.W., Schwarze V.S. (eds) Geodesy-The Challenge of the 3rd Millennium. Springer, Berlin, Heidelberg, doi.org/10.1007/978-3-662-05296-9_19
Heiskanen W.A., Moritz H., 1967, Physical Geodesy, W.H.Freeman, San Francisco

Hwang C., 1998, Inverse Vening Meinesz formula and deflectiongeoid formula: applications to the predictions of gravity and geoid over the South China Sea. Journal of Geodesy, 72, 304312.

Miao L., Heiner D. and Jürgen M., Regional gravity field modeling using free-positioned point masses. Studia Geophysica et Geodaetica. 2014

Moritz H., 1980, Advanced Physical Geodesy. Herbert Wichmann Verlag, Karlsruhe

Moritz H., 1966. Linear Solutions of the Geodetic Boundary-Value Problem, Dept. of Geodetic Science and Surveying, the Ohio State University, Columbus

Wenzel H. G., 2005, Global models of the gravity field of high and ultra-high resolution, [International School for the Determination and Use of the Geoid], Budapest, Hungary. 